\title{
Qualitative Real-Time Range Extraction for Preplanned Scene Partitioning Using Laser Beam Coding
}

\author{
Didi Sazbon ${ }^{1}$, Zeev Zalevsky ${ }^{2}$, and Ehud Rivlin ${ }^{1}$ \\ ${ }^{1}$ Department of Computer Science, Technion - Israel Institute of Technology, Haifa, Israel \\ ${ }^{2}$ School of Engineering, Bar-Ilan University, Ramat-Gan, Israel
}

\begin{abstract}
This paper proposes a novel technique to extract range using a phaseonly filter for a laser beam. The workspace is partitioned according to $M$ meaningful preplanned range segments, each representing a relevant range segment in the scene. The phase-only filter codes the laser beam into $M$ different diffraction patterns, corresponding to the predetermined range of each segment. Once the scene is illuminated by the coded beam, each plane in it would irradiate in a pattern corresponding to its range from the light source. Thus, range can be extracted at acquisition time. This technique has proven to be very efficient for qualitative real-time range extraction, and is mostly appropriate to handle mobile robot applications where a scene could be partitioned into a set of meaningful ranges, such as obstacle detection and docking. The hardware consists of a laser beam, a lens, a filter, and a camera, implying a simple and cost-effective technique.
\end{abstract}

\section{Introduction}

Range estimation is a basic requisite in Computer Vision, and thus, laser techniques utilizing pattern light have been explored to a great extent. Pattern light is commonly used in a stereo configuration in order to facilitate the correspondence procedure, which forms the challenging part of triangulation. Usually, one camera is replaced by a device that projects pattern light (also known as 'structure light'), while the scene is grabbed by the other camera. A very popular group of techniques are known as 'coded structured light'. The coding is achieved either by projecting a single pattern or a set of patterns. The main idea is that the patterns are designed in such a way that each pixel is assigned with a codeword [1]. There is a direct mapping between the codeword of a specific pixel and its corresponding coordinates, so correspondence becomes trivial. Different types of patterns are used for the coding process, such as: black and white, gray scale, and RGB [2-7]. Coded structure light is considered one of the most reliable techniques for estimating range, but since usually a set of patterns is needed, it is not applicable to dynamic scenes. When using only one pattern, dynamic scenes might be allowed, but the results are usually of poor resolution.

Additional techniques implementing structured light to assist the correspondence procedure include sinusoidal varying intensities, stripes of different types (e.g. colored, cut), and projected grids [8-15]. These methods, although projecting only one pattern, still exploit a time consuming search procedure. 
Here, pattern light is used only with one image to directly estimate range. No correspondence (triangulation) is needed, and the setup consists only of a laser beam, a lens, a single mask, and a camera. The main concept would be to partition the workspace into a set of range segments, in a way that would be meaningful for a working mobile robot. The motivation lies in the fact that in order to perform tasks such as obstacle detection or docking, it should be sufficient that the robot would be able to distinguish between a set of predefined ranges. The idea is to code a laser beam into different patterns, where each pattern corresponds to a specific range segment. Once a scene is illuminated by the coded beam, each patch in it would irradiate with the pattern that corresponds to its range from the light source. The beam coding is merely realized by one special phase-only filter, and consequently, the technique is accurate, fast (hardware solution), cost-effective, and in addition, fits dynamic scenes.

\section{Qualitative Real-Time Range Extraction for Preplanned Scene Partitioning Using Laser Beam Coding}

The proposed technique is based on an iterative design of a phase-only filter for a laser beam. The relevant range is divided into $M$ meaningful planes. Each plane, once illuminated by a laser beam that propagates through the phase-only filter, would irradiate in a different, predetermined, pattern. The pattern that was chosen here consists of gratings in $M$ different angles (slits). Each range would be assigned with slits having a unique angle. Once a plane is illuminated, it would irradiate with the angular slits pattern that is proportional to its range.

The iterative procedure is based on the Gerchberg-Saxton (GS) algorithm [16] as schematically illustrated in Figure 1. What follows is a description of the general concept of the algorithm. Assume we have a function denoted by $f(x, y)$, then, $f(x, y)$ could be represented as:

$$
f(x, y)=|f(x, y)| \cdot \exp \{i \cdot \phi(x, y)\}
$$

where, $|f(x, y)|$ is the amplitude of $f(x, y)$, and $\phi(x, y)$ is the phase of $f(x, y)$. We would denote the Fourier Transform of $f(x, y)$ by $F(u, v)$, thus:

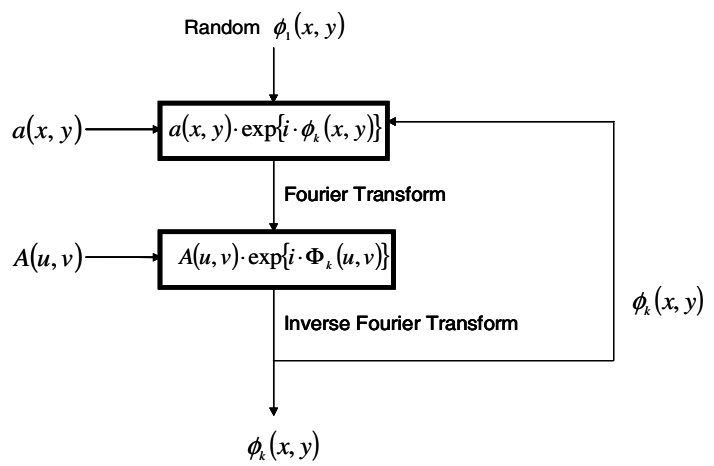

Fig. 1. A schematic description of the GS algorithm to obtain phase information 


$$
F(u, v)=|F(u, v)| \cdot \exp \{i \cdot \Phi(u, v)\}
$$

where, $|F(u, v)|$ is the amplitude of $F(u, v)$, and $\Phi(u, v)$ is the phase of $F(u, v)$. Assume $|f(x, y)|$ and $|F(u, v)|$ are determined in advance and are denoted by $a(x, y)$ and $A(u, v)$, accordingly. In order to retrieve the phase, $\phi(x, y)$, from $f(x, y)$, we start with a random estimation of $\phi(x, y)$, denoted by $\phi_{1}(x, y)$. Thus, $f(x, y)$ is estimated by: $a(x, y) \cdot \exp \left\{i \cdot \phi_{1}(x, y)\right\}$. In order to design a phase-only filter, such that, using a laser beam would result in a predefined pattern, we would use $a(x, y)=1$, and $A(u, v)$ will be a function that depicts the desired pattern in which the beam, while propagating in free space, would illuminate. Although not proven mathematically, the algorithm is known to give excellent practical results.

Here, we would like to use that concept, but to create a phase-only filter that would illuminate in a pattern, that slightly changes, as a function of range. Thus, the GS algorithm should be modified to comply with these changes. The modified procedure is as follows: let $a(x, y)=1$, let $Z^{j}(u, v)$ be the pattern assigned to the $j$-th plane $(j=1,2, \ldots, M)$, and start with $\phi_{1}(x, y)$, a random estimation of $\phi(x, y)$. Proceed with the iterative procedure that is depicted schematically in Figure 2.

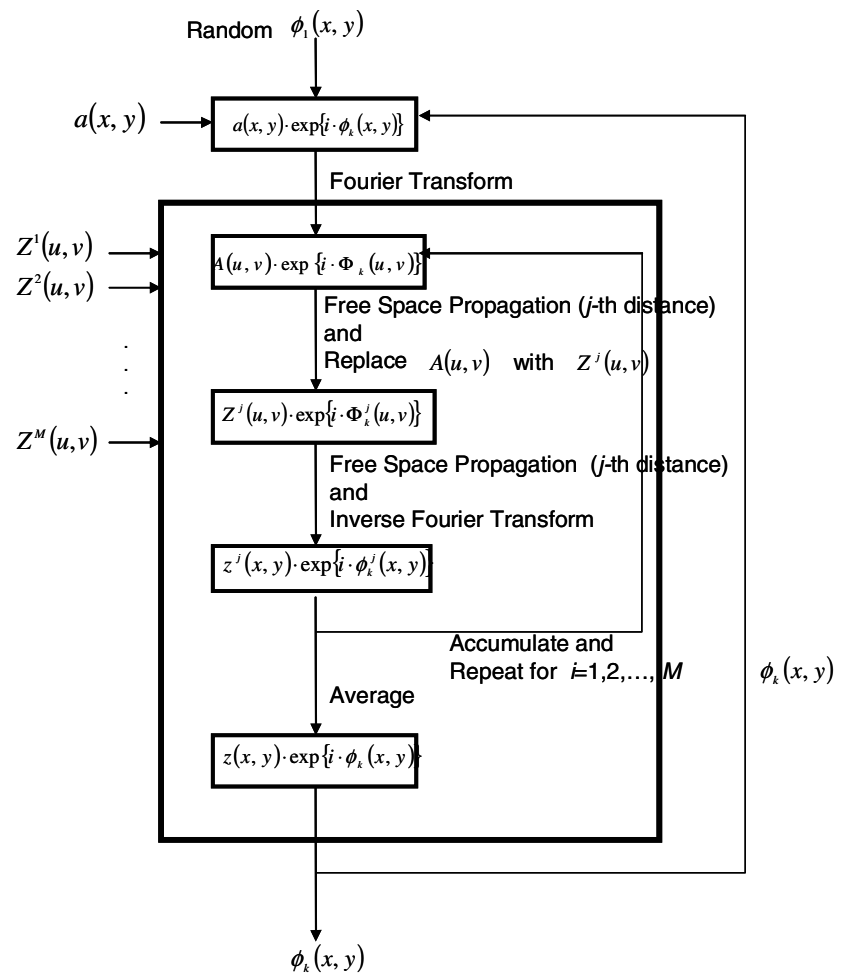

Fig. 2. A schematic description of the algorithm presented here 
Note the term Free Space Propagation used along the procedure (Figure 2). The laser beam is propagated to the position of the plane $Z^{j}(u, v)$ by multiplying its spatial spectrum using the term:

$$
F S\left(x, y, d_{j}\right)=\exp \left[\frac{2 \pi i \cdot d_{j}}{\lambda} \sqrt{1-\left(\lambda \frac{x}{D}\right)^{2}-\left(\lambda \frac{y}{D}\right)^{2}}\right]
$$

where, $d_{j}$ is the range of the plane from the origin, $\lambda$ is the wave length, and $D \times D$ are the dimensions (in meters) of the detected plane. Note also, that the planes parameters (i.e. the number of planes, the size of a plane, the location of a plane, the distances between planes - that could vary, and the patterns to be displayed on the planes) can be defined to meet specific requirements of the phase-only filter.

The expected behavior of the laser beam once illuminated and according to the physical characteristics of it would be as follows. The beam would be homogeneous until it propagates and encounters the first predefined plane, then it would exhibit the first designed slit pattern. It would keep the same pattern while propagating along the first segment until encountering the second predefined plane, then it would exhibit the second designed slit pattern. It would keep the same pattern while propagating along the second segment and so on. When it would meet the last predefined plane, it would keep propagating indefinitely with its corresponding slit pattern.

Note that the range segments can differ in length and the partitioning should not necessarily be uniform. For example, a docking mobile robot would like to decelerate first at 30 meters from the target, then at 5 meters, and again at 1 meter. The resultant phase-only filter would consist of 3 slits patterns, corresponding to the range segments of 1,5 , and 30 meters. Thus, each filter should be designed with range segments that meet the needs of the relevant task, the specific working robot, and the particular workspace.

\section{Results}

The proposed technique was tested with a phase-only filter designed to exhibit the patterns depicted by Figure 3, on six equally spaced planes positioned between 0.5 to 1 meters from the light source. The range between two consecutive planes equals to 0.1 meters. A laser beam having a wave length of $0.5 \cdot 10^{-6}$ meters (green light) was used. The physical size of the filter is $4 \times 4$ millimeters, and the beam was scattered in order to cover the whole filter. By using the technique described in Section 2, the

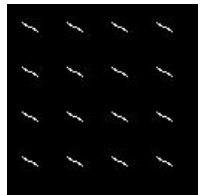

(a)

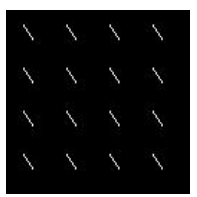

(b)

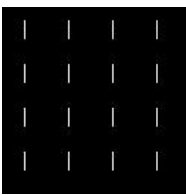

(c)

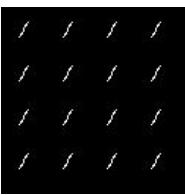

(d)

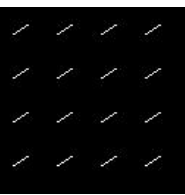

(e)

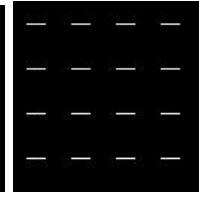

(f)

Fig. 3. The slits patterns used here 
resulted filter has values in the range $[-\pi, \pi]$. In order to save in production costs, the filter was quantized into two levels: $-\pi$ and 0 . As can be seen throughout the experiments, the results are satisfying, while the production is extremely cost effective.

Figure 4 shows images depicting the patterns irradiated by the phase-only filter on planes positioned at ranges $0.5,0.6,0.7,0.8,0.9$, and 1 meter from the light source. Figure 5 shows the neighborhood of the patterns and the slits directions are clearly visible to the human eye. In order to automate the application and deduce the range from the assignment of a specific direction to a particular image a simple procedure can be invoked. Since these images were taken from a non calibrated camera they need simple preprocessing.

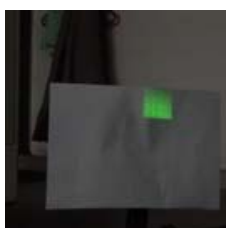

(a)

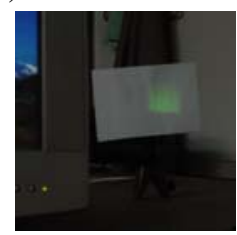

(d)

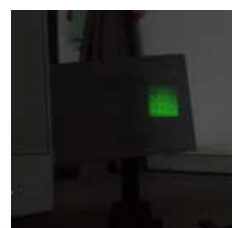

(b)

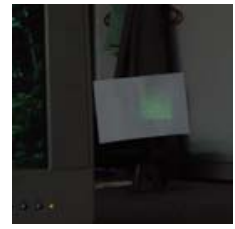

(e)

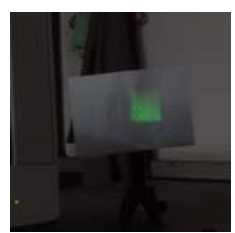

(c)

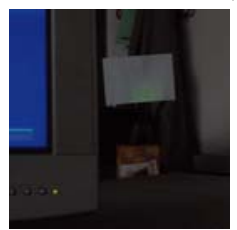

(f)

Fig. 4. The patterns irradiated by using the phase only filter at different ranges

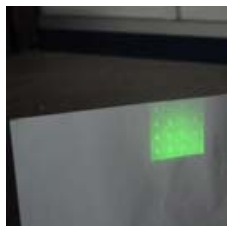

(a)

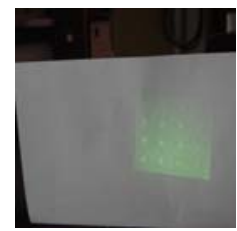

(d)

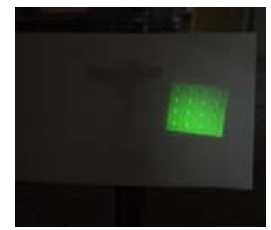

(b)

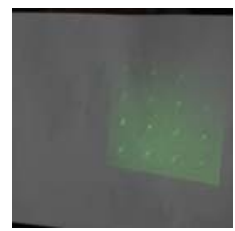

(e)

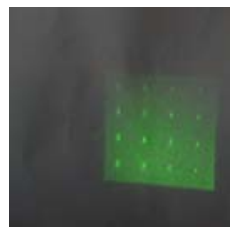

(c)

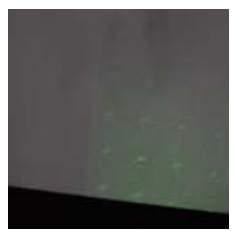

(f)

Fig. 5. The patterns of Figure 4 in closer look depicting better the slits directions

The first step consists of rotating the images, so their patterns would be aligned horizontally, and normalizing their color, so they would be comparable with the correlation patterns. The correlation patterns are merely images of the six possible slits. 
Then in the next step, taking into considerations the fact that the laser is of bright green color that pops up in the images, a simple threshold is applied leaving only the relevant information. The third step is to correlate the image with the six possible patterns (i.e. slits in different directions) to get maximum response on the most compatible one. Applying this procedure confirmed that maximum correlation values corresponded to the expected patterns.

The results clearly demonstrate that using the proposed technique, range is determined immediately, in real time. In addition to its accuracy, simplicity, and speed, the technique is extremely cost effective, it comprises only of a laser beam, a lens, a filter, and a common camera.

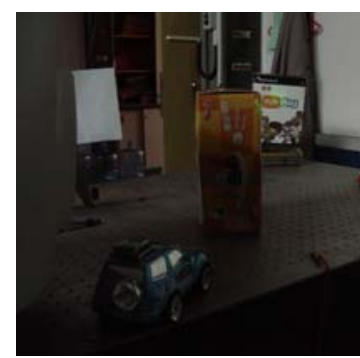

(a)

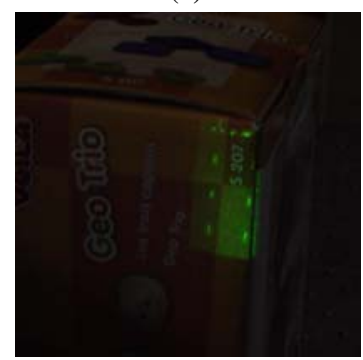

(c)

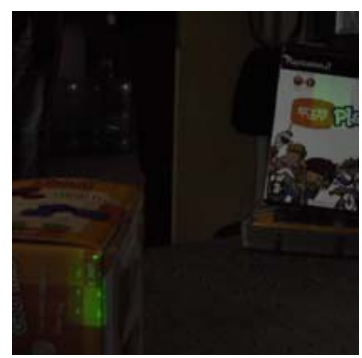

(b)

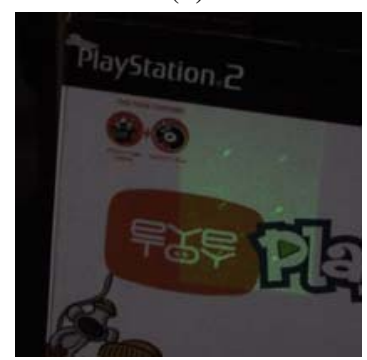

(d)

Fig. 6. A semi-realistic scene

The accuracy of the technique was measured at the border planes where the patterns were supposed to change, and found out that all the ranges were accurate up to 1-3 millimeters independently from the range itself. This implies that this specific filter has a reliability of $97 \%$ in finding the border ranges. Also, if a robot would be interested in finding out when it is exactly on a border plane, it only needs to find a point of change between two successive patterns. Considering the directions of the two patterns, the range is directly deduced. Note that, in general, if a different filter would be designed its accuracy and reliability should be measured individually.

Figure 6a depicts a semi-realistic scene where a mobile robot (the car) illuminates the scene using the proposed phase-only filter in order to detect obstacles. Two boxes, acting as obstacles, are positioned in front of it, and it can be clearly seen that the pattern of the filter is split between both of them, where one half irradiates in a specific pattern and the other half irradiates in a different pattern. For a better observation, Figure $6 \mathrm{~b}$ consists of a closer image of the obstacles, while Figure $6 \mathrm{c}$ and $6 \mathrm{~d}$ 
consist of even closer images of each of the obstacles. By analyzing the patterns it can be deduced that the first obstacle is located at distance between 0.7 and 0.8 meters and the second at distance between 0.8 and 0.9 meters (in respect to the light source).

\section{Discussion}

A technique to qualitative real-time range estimation for preplanned scene partitioning is presented here. The setup consists of a laser beam, a lens, a single phase-only filter, and a camera. The phase-only filter is designed in such a way, that a scene patch illuminated by it, would irradiate in a unique pattern proportional to its range from the light source. The phase-only filter can be designed to meet the specific parameters of its working environment. Relevant parameter include: the location (range) of the first range segment, the number of range segments, the length of each segment (e.g. shorter for nearby environment), the uniformity of the gaps (e.g. equal, changing), the dimensions of the projected pattern (e.g. 10 centimeters, 1/2 meter), and the density of the slits forming the pattern. If the environmental conditions require a stronger contrast, a stronger laser source can be used. Note, since the physics of propagating light should be taken into considerations, the dimensions of the projected pattern are getting bigger as the range increase.

The specific scanner implemented here and described in the Results Section, is in fact, a very simple one. It could be assembled using available laboratory components. Thus, its main role in proving the correctness of the technique, and as such it was designed having a relatively short total range (0.5-1 meter) with relatively long range segments (0.1 meter), best suitable for the task of obstacle detection or docking. The environmental factors that might affect the accuracy or the reliability of this scanner are light conditioning or green obstacles. If the light is too strong, the green slits can be hardly seen. Also, if the scene would consist of green obstacles, it might be difficult to separate the slits from the background. This problem, when appropriate, can be resolved by using a laser beam of red light.

In general, the technique would mostly fit in a context of a mobile robot that would be interested in a rough estimation of a scene structure. This would enable it to identify guidelines in predetermined ranges and consequently, plan its path. The workspace can be partitioned in advance into a set of relevant ranges composed of near, intermediate, and far at the same time, with variable length of segments. Near ranges would naturally be densely segmented, whereas far ranges would be segmented in sparse manner. The robot would have its range partitioned into an appropriate and meaningful warning zones, so when a match is achieved, a corresponding action could be invoked. The technique extremely fits such scenarios by providing both qualitative and reliable results.

\section{References}

1. Salvi, J., Pages, J., Batlle, J.: Pattern Codification Strategies in Structured Light Systems. Pattern Recognition Vol. 37. (2004) 827-849

2. Caspi, D., Kiryati, N., Shamir, J.: Range Imaging with Adaptive Color Structured Light. IEEE Transactions on PAMI Vol. 20, No.5. (1998) 470-480 
3. Horn, E., and Kiryati, N.: Toward Optimal Structured Light Patterns. Image and Vision Computing Vol. 17, No. 2. (1999) 87-97

4. Manabe, Y., Parkkinen, J., Jaaskelainen, T., Chihara, K.: Three Dimensional Measurement Using Color Structured Patterns and Imaging Spectrograph. ICPR'02 Vol. 3. (2002) 649-652

5. Pages, J., Salvi, J., Matabosch, C.: Implementation of a Robust Coded Structured Light Technique for Dynamic 3D Measurements. ICIP'03 Vol. 3. (2003) 1073-1076

6. Sato, K., Inokuchi, S.: Range-Imaging System Utilizing Nematic Liquid Crystal Mask. ICCV'87. (1987) 657-661

7. Valkenburg, R.J., McIvor, A.M.: Accurate 3D Measurement Using a Structured Light System. Image Vision and Computing Vol. 16. (1998) 99-110

8. Albamont, J., Goshtasby, A.: A Range Scanner with a Virtual Laser. Image and Vision Computing Vol. 21. (2003) 271-284

9. Fofi, D., Salvi, J., Mouaddib, E.M.: Uncalibrated Reconstruction: an Adaptation to Structured Light Vision. Pattern Recognition Vol. 36. (2003) 1631-1644

10. Furukawa, R., Kawasaki, H.: Interactive Shape Acquisition Using Marker Attached Laser Projector. 3DIM'03. (2003) 491-498

11. Guisser, L., Payrissat, R., Castan, S.: PGSD: an Accurate 3D Vision System Using a Projected Grid for Surface Descriptions. Image and Vision Computing Vol. 18. (2000) 463-491

12. Je, C., Lee, S.W., Park, R.-H.: High-Contrast Color-Stripe Pattern for Rapid StructuredLight Range Imaging. ECCV'04 Vol. 1. (2004) 95-107

13. Kang, S.B., Webb, J.A., Zitnick, C., Kanade, T.: A Multibaseline Stereo System with Active Illumination and Real-Time Image Acquisition. ICCV'95. (1995) 88-93

14. Maruyama, M., Abe, S.: Range Sensing by Projecting Multiple Slits with Random Cuts. IEEE Transactions on PAMI Vol. 15, No. 6. (1993) 647-651

15. Scharstein, D., Szeliski, R.: High-Accuracy Stereo Depth Maps Using Structured Light. CVPR'03 Vol. 1. (2003) 195-202

16. Gerchberg, R.W., Saxton, W.O.: A Practical Algorithm for the Determination of Phase from Image and Diffraction Plane Pictures. Optik Vol. 35. (1972) 237-246 